\title{
A REMARK ON KNOT GROUPS WITH A CENTER
}

\section{LEE P. NEUWIRTH}

Introduction. In [1] it is conjectured that the groups of torus knots are the only groups of tame nontrivial knots in $S^{3}$ which have a nontrivial center. Herein this problem is reduced to studying the centers of knot groups whose commutator subgroups are finitely generated. Two immediate bonuses accrue from the theorem proved. The first is the proof of the above conjecture for genus one knots and the second is a stringent condition on the Alexander polynomial of a knot group with a center.

The notation used is that of [1].

\section{Main Theorem.}

THEOREM. If a knot group $G$ has a nontrivial center, then $[G, G]$ is free of finite rank.

Proof. Recall that in [1] it is proved that $[G, G]$ may take one of three forms. One form satisfies the conclusion of the theorem. The other two will be shown to be impossible.

According to [1] the center of $G, Z(G)$ does not intersect $[G, G]$, consequently the abelianizing homomorphism maps $Z(G)$ isomorphically onto a subgroup of $Z=(t:)$. Let $t^{r}$ denote a generator of this subgroup. Since $Z$ is a free group, $G$ splits and every element may be written in the form $t^{s} C$, where $C \in[G, G]$. Let $t^{r} C$ denote a generator of $Z(G)$. Then $\left(t^{r} C\right) X\left(t^{r} C\right)^{-1}=X$ so that

$$
t^{-r} X t^{r}=C X C^{-1},
$$

for all $X \in G$.

Suppose now that $[G, G]$ has the form

$$
\cdots \underset{F_{2 \sigma}}{*} A_{-1} \underset{F_{2 \sigma}}{*} A_{F_{2 \sigma}} * A_{F_{2 \sigma}} A_{1} * \cdots
$$

We may suppose without loss of generality that

$$
C \in A_{\infty}=\underset{F_{2 \sigma}}{A_{0} *} A_{F_{2 \sigma}} * \cdots
$$

(recall each factor of a free product with amalgamation is a subgroup of that product). Let $X$ denote an element of $A_{0}$ which is not in $A_{0} \cap A_{1}$.

Now the action of $t$ on $[G, G]$ is to map each $A_{i}$ onto $A_{i+1}$, so that

Received by the editors April 11, 1962. 
$t^{-r} X t^{r}$ lies in $A_{-r}$, and since $X \notin A_{0} \cap A_{1}, \quad\left(t^{-r} X t^{r}\right) \notin A_{-r} \cap A_{-r+1}$; it follows then that $\left(t^{-r} X t^{r}\right) \notin A_{-1} \cap A_{0}$ hence $t^{-r} X t^{r} \notin A_{\infty}$, but $C X C^{-1}$ $\in A_{\infty}$ so that (1) cannot be true. This dispenses with possibility $\mathrm{A}$ in Theorem 1 of [1]. Case $\mathrm{B}$ is treated similarly as follows: If $[G, G]$ $=A_{0} \subset A_{1} \subset A_{2} \subset \cdots$ and $C \in A_{k}$, then pick $X \in A_{k}, X \notin A_{k-1}$ then $\left(t^{r} X t^{-r}\right) \in A_{k+r},\left(t^{r} X t^{-r}\right) \notin A_{k+r-1}$ but $C X C^{-1} \in A_{k}$ and since $r>0$ equation (1) is impossible. This completes the proof of the theorem.

Corollaries. For knot groups whose commutator subgroups are free, the Alexander matrix is simply $M-t I$ where $M$ is a unimodular integer matrix which describes the automorphism of

$$
[G, G] /[[G, G],[G, G]]
$$

induced by a generator $t$ of $G /[G, G]$ and $I$ is the identity matrix. Since $Z(G) \cap[G, G]=1$ it follows that if $Z(G) \neq 1$, some power, say $r$, of $t$ leaves

$$
[G, G] /[[G, G],[G, G]]
$$

fixed, so that $M^{r}$ is $I$, hence every characteristic root of $M$ is an $r$ th root of unity. But $|M-t I|$ is just the Alexander Polynomial, thus we may state:

COROLlaRY 1. If a knot group has a center, every root of the Alexander Polynomial is an rth root of unity.

COROLlARY 2. If a knot of genus 1 has a center, its group is that of the trefoil knot.

Proof. By Theorem 1 the commutator subgroup is free of finite rank; by [1], since the genus is 1 , this rank must be 2 ; by [2] the only knot groups with this property are $3_{1}$ and $4_{1}$; by Corollary 1 and computation, $4_{1}$ cannot have a center. q.e.d.

\section{BIBLIOGRAPHY}

1. L. Neuwirth, On the algebraic determination of the genus of knots, Amer. J. Math. 82 (1960), 791-798.

2. E. S. Rapaport, On the commutator subgroup of a knot group, Ann. of Math. (2) 71 (1960), 157-162.

INSTITUTE For DefEnse ANALyses 